\title{
Targeted lipid biomarker screening in the lower oceanic crust guided by gene expression analyses - new avenues for the detection of biosignatures in ultra- low biomass samples
}

FLORENCE SCHUBOTZ ${ }^{1}$, PARASKEVI MARA ${ }^{2}$, JULIUS SEBASTIAN LIPP ${ }^{1}$, LARA A.E. MEYER ${ }^{1,3}$, MAXENCE QUÉMENER $^{4}$, GAËTAN BURGAUD ${ }^{4}$, JIANGTAO LI ${ }^{5}$ AND VIRGINIA P. EDGCOMB ${ }^{2}$

${ }^{1}$ MARUM, University of Bremen

${ }^{2}$ Woods Hole Oceanographic Institution

${ }^{3} \mathrm{ETH}$

${ }^{4}$ Université de Brest

${ }^{5}$ Tongi University

Presenting Author: schubotz@uni-bremen.de

Untargeted, broad lipidomic screening of ultra-low biomass samples from nutrient-depleted habitats such as the oligotrophic ocean or the deep biosphere is frequently hampered by low in situ biomass. Targeted lipid analyses using multiple reaction monitoring (MRM) mass spectrometry can be used to overcome this challenge. However, specific biomarkers must be selected from out of the myriad potential structural combinations that exist, particularly for bacterial and eukaryotic lipids. Typically, such targeted screening methods are guided by the lipid composition of successfully cultured microbial strains isolated from these environments, however the lipid composition (head group and side chain diversity) of cultured microbial strains might differ from microbes that live in these habitats. Here, we applied a new method to lipid biomarker analyses that uses gene expression data to target lipid biomarkers of interest. Metatranscriptome data from the lower oceanic crust, where access to circulating fluids along cracks and fissures enables microbial life to persist [1], were searched for genes involved in lipid synthesis, and these were used to guide lipid biomarker selections. Information from lipid analyses of fungal cultures isolated from the same samples contributed additional information [2]. Surprisingly, many genes for generic phospholipid biosynthesis were not detected in the metatranscriptomes, and neither were genes related to the biosynthesis of lipids identified in the fungal cultures. Instead, expressed genes involved in the biosynthesis of cardiolipin, a lipid often invoked during stationary growth and cell survival [3] as well glycolipid headgroups and ether-bound core lipids, which have been suggested to reduce cellular maintenance, were detected. The metatranscriptome data also confirmed our initial detection of core diether glycerol lipids in these samples [1]. We are currently implementing the MRM technique for lower ocean crust samples and envision this approach will open promising new avenues for low-biomass lipid detection and for lipid assignments to distinct groups of active organisms.

[1] Li et al. (2020), Nature, 579, 250-255.

[2] Quémener et al. (2020), EM, 22, 3950-3967.

[3] Hiraoka et al. (1993), FEBS Lett, 336, 221-224. 